\title{
Vapor-solid interfacial reaction and polymerization for wafer-scale uniform and ultrathin two-dimensional organic films
}

\author{
Wenqian $\mathrm{Yao}^{1,2 \dagger}$, He Yang ${ }^{1 \dagger}$, Qingsong Zhang ${ }^{1}$, Longxian Shi ${ }^{1,2}$, Jianzhe Sun ${ }^{1}$, Yunlong Guo ${ }^{1}$, Lang Jiang ${ }^{1}$, \\ Bin $\mathrm{Wu}^{1 *}$ and Yunqi Liu ${ }^{1,2^{*}}$
}

\begin{abstract}
Chemical vapor deposition is a conventional synthesis method for growing large-scale and high-quality two-dimensional materials, such as graphene, hexagonal boron nitride, and transition-metal dichalcogenides. For organic films, solution-based methods, such as inkjet printing, spin coating, and drop and micro-contact printing, are commonly used. Herein, we demonstrate a general method for growing wafer-scale continuous, uniform, and ultrathin $(2-5 \mathrm{~nm})$ organic films. This method is based on a copper $(\mathrm{Cu})$ surfacemediated reaction and polymerization of several equivalent bromine $(\mathrm{Br})$-containing $\pi$-conjugated small molecules $\left(\mathrm{C}_{12} \mathrm{~S}_{3} \mathrm{Br}_{6}, \mathrm{C}_{24} \mathrm{H}_{4} \mathrm{O}_{2} \mathrm{Br}_{2}\right.$, and $\left.\mathrm{C}_{24} \mathrm{H}_{12} \mathrm{Br}_{2} \mathrm{~N}_{4}\right)$, in which local surface-mediated polymerization and internal $\pi-\pi$ interactions among organic molecules are responsible for the dimension and uniformity control of the thin films. Specifically, the growth rate and morphology of thin films were found to be $\mathrm{Cu}$-facet-dependent, and single-crystal $\mathrm{Cu}(111)$ surfaces could improve the uniformity of thin films. In addition, the number of Br groups and size of organic molecules were critical for crystallinity and thin-film formation. This method can be used to fabricate heterostructures, such as organic film/graphene, giving room for various functional materials and device applications.
\end{abstract}

Keywords: organic films, interface, chemical vapor deposition, copper catalyst, debromination

\section{INTRODUCTION}

Manipulating the condensed state of materials is critical for their properties and various applications. Compared with inorganic materials, the building blocks of organic materials usually have much larger sizes, various geometric shapes, and flexible functional groups in terms of types, numbers, and positions [1-9]. These degrees of freedom provide morphological, structural, and dimensional flexibility for organic materials; however, it is difficult to control such parameters.

Various methods, including physical vapor deposition and solution- or interface-based methods, have been employed in fabricating low-dimensional organic materials such as individual crystals at nanometer or micrometer length scale [10-14], or two-dimensional (2D) organic thin films, which are important for many applications, including organic field-effect-transistors $[5,8]$ and organic light-emitting diodes [9]. Generally, these methods can be grouped into two in terms of the interactions involved in the process. In the first type, the driving force for organizing materials arises mainly from $\pi-\pi$ van der Waals interactions among conjugated organic molecules. The second type takes advantage of both $\pi-\pi$ and external interactions, such as mechanical forces [15] and capillary force [16]. The former is governed in the case of physical vapor deposition. Through this approach, several specific conjugated molecules with rod or disk shapes, such as $\alpha$-sexithiophene, pentacene, dioctylbenzothienobenzothiophene ( $\mathrm{C}_{8}$-BTBT), and metal phthalocyanine, have been grown into crystalline ultrathin thin films with a monolayer to few layers on inert surfaces, such as $\mathrm{SiO}_{2}$, mica, and graphene [17-19]. However, due to the lack of control over interactions between substrates and organic molecules, this method usually limits material crystallinity to the nanometer or micrometer length scale, and events of well-organized 2D conformation of organic molecules, which is highly dependent on the special properties of molecules, are rarely observed.

The latter can be found in various cases, such as inkjet printing, spin coating, drop printing, bar-coating, and microcontact printing [12]. Thus, the lack of control over the interactions between substrates and the formed organic crystal causes the dispersion of discrete crystals on inert surfaces, and various external forces involved in the latter case provide a global effect on controlling the arrangement of small organic molecules and the dimension of the final product, which causes local nonuniformity and discontinuity of the as-prepared thin films [2026]. In principle, there is a large room for introducing new interactions between substrates and organic molecules with a local effect that can fill the weak to strong interaction gap, improving the control of organic thin-film fabrication and the understanding of the relationships between organic molecular structures and the grown films.

Herein, we found that copper $(\mathrm{Cu})$ surface-mediated polymerization of several bromine $(\mathrm{Br})$-containing $\pi$-conjugated small molecules is effective for growing continuous, uniform, and ultrathin $(2-5 \mathrm{~nm})$ polycrystalline films. The local surfacemediated polymerization effect and internal $\pi-\pi$ interactions

\footnotetext{
${ }^{1}$ Beijing National Laboratory for Molecular Sciences, Key Laboratory of Organic Solids, Institute of Chemistry, Chinese Academy of Sciences, Beijing 100190, China

${ }^{2}$ Sino-Danish Center for Education and Research, Sino-Danish College, University of Chinese Academy of Sciences, Beijing 100049, China

† These authors contributed equally to this work.

* Corresponding authors (emails: wubin@iccas.ac.cn (Wu B); liuyq@iccas.ac.cn (Liu Y))
} 
among organic molecules are responsible for the dimension and uniformity controls of thin films. In particular, the growth rate and morphology of thin films depend on $\mathrm{Cu}$ facets, and a singlecrystal $\mathrm{Cu}(111)$ surface could improve the uniformity of thin films. We also found that the number of $\mathrm{Br}$ groups and the size of organic molecules are critical for the crystallinity and formation of thin films. As-grown films from $\mathrm{C}_{12} \mathrm{~S}_{3} \mathrm{Br}_{6}$ exhibited hole mobility of $10^{-4} \mathrm{~cm}^{2} \mathrm{~V}^{-1} \mathrm{~s}^{-1}$ and an on-off current ratio of approximately 94 . This method can be readily used to fabricate heterostructures, such as organic film/graphene.

\section{EXPERIMENTAL SECTION}

\section{Synthesis of organic $\mathrm{C}_{12} \mathrm{~S}_{3} \mathrm{Br}_{6}$ molecules}

Hexabromobenzo[1,2-b:3,4- $\left.b^{\prime}: 5,6-b^{\prime \prime}\right]$ trithiophene (HBBTT) was synthesized from benzo[1,2-b:3,4- $\left.b^{\prime}: 5,6-b^{\prime \prime}\right]$ trithiophene (BTT) (Scheme 1). Bromine $(4.4 \mathrm{~mL}, 85 \mathrm{mmol}$ ) was added dropwise in a solution of BTT $(2.44 \mathrm{~g}, 9.91 \mathrm{mmol})$ in nitrobenzene $(80 \mathrm{~mL})$ with iron powder $(0.18 \mathrm{~g}, 3.6 \mathrm{mmol})$ for $5 \mathrm{~min}$. The solution was allowed to stand for $16 \mathrm{~h}$ and was heated under reflux at $180^{\circ} \mathrm{C}$ for $2 \mathrm{~h}$. Using $-20^{\circ} \mathrm{C}$ ethanol as a reflux coolant, the mixture was cooled to room temperature, washed with diethyl ether $(300 \mathrm{~mL})$, and filtered to yield $6.42 \mathrm{~g}(90 \%)$ of white powders.

\section{Growth of fibers and films of organic materials}

The experiments were conducted using a chemical vapor deposition (CVD) system with a 2 -inch quartz tube. First, 300nm-thick $\mathrm{SiO}_{2} / \mathrm{Si}$, polycrystalline $\mathrm{Cu}$, and single-crystal $\mathrm{Cu}(111)$ foils were used as substrates placed at the center of the furnace Zone 2, and several Br-containing conjugated molecules were separately used as the sources placed on Zone 1 (Fig. 1a). Once substrates and sources were placed at the desired locations, the temperatures of the two zones were raised to the desired values with the flow of argon (Ar) and hydrogen gas $\left(\mathrm{H}_{2}\right)$. After a suitable time of growth, the furnace was switched off and cooled to room temperature. In the case of organic film/graphene heterostructures, graphene grown on $\mathrm{Cu}$ surface was used as substrates, and the growth conditions are similar to the above.

\section{Transfer of samples to $\mathrm{SiO}_{2} / \mathrm{Si}$ substrate and transmission electron microscopy (TEM) grid}

Typically, a thin layer of poly(methyl-methacrylate) (PMMA) was spin-coated onto the sample at $3000 \mathrm{r} \mathrm{min}^{-1}$ and baked at $120^{\circ} \mathrm{C}$ for $10 \mathrm{~min}$. The $\mathrm{H}_{2}$ bubbling process was conducted in an electrolytic cell with a PMMA-coated substrate as the cathode and a platinum foil as the anode. $\mathrm{A} \mathrm{NaOH}$ aqueous solution $\left(0.5 \mathrm{~mol} \mathrm{~L}^{-1}\right)$ was used as the electrolyte. After complete separation from the $\mathrm{Cu}$ substrate, the PMMA-coated film was collected and rinsed in deionized water, and the film was placed onto a $\mathrm{SiO}_{2} / \mathrm{Si}$ substrate or TEM grid. Afterward, the sample was baked at $80^{\circ} \mathrm{C}$ for $5 \mathrm{~min}$ to remove water residue before the PMMA was dissolved by acetone.

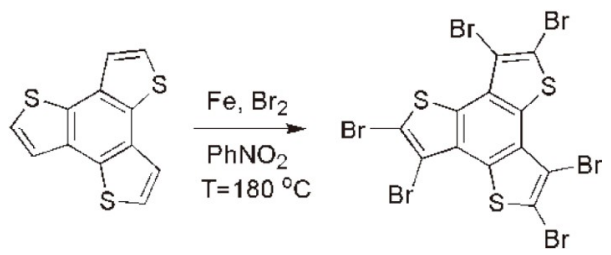

Scheme 1 The synthesis protocol of HBBTT.

\section{Characterizations}

Samples were characterized by optical microscopy (Olympus DX51, Olympus), fluorescent microscopy (Olympus IX83 with an excitation wavelength of $325-375 \mathrm{~nm}$ ), X-ray diffraction (XRD; PANalytical Empyrean diffractometer using $\mathrm{Cu}$ radiation), X-ray photoelectron spectroscopy (XPS; ESCALAB250XI), thermogravimetric analysis (TGA; PerkinElmer Diamond TG/ DTA), Raman spectroscopy with an excitation wavelength of $532 \mathrm{~nm}$ (Renishaw inVia, Renishaw), atomic force microscopy (AFM) with Veeco Nanoscope IIIa, tapping mode, and TEM by JEOL 2010F. Electrical measurements were performed using a semiconductor characterization system (Keithley 4200-SCS). The mobility was calculated using the following equation:

$\mu=\frac{L}{C_{\mathrm{g}} V_{\mathrm{d}} W} \times \frac{\mathrm{d} I_{\mathrm{d}}}{\mathrm{d} V_{\mathrm{g}}}$,

where $V_{\mathrm{d}}$ and $V_{\mathrm{g}}$ are the source-drain and gate voltage, respectively; $L$ and $W$ are the channel length and width, respectively; $C_{\mathrm{g}}\left(11 \mathrm{nF} \mathrm{cm}^{-2}\right)$ is the capacitance of the back-gate dielectric layer; $I_{\mathrm{d}}$ is the source-drain current.

\section{RESULTS AND DISCUSSION}

A new small conjugated molecule $\mathrm{C}_{12} \mathrm{~S}_{3} \mathrm{Br}_{6}$ was used as the starting material (see EXPERIMENTAL SECTION). A common physical vapor-deposition method was employed to control the aggregation state of small molecules (Fig. 1a). TGA showed that the molecules are stable below $340^{\circ} \mathrm{C}$ (Fig. S1). Maintaining the temperature of source Zone 1 at $280^{\circ} \mathrm{C}$ and collecting Zone 2 at $180^{\circ} \mathrm{C}$, the conjugated molecule, $\mathrm{C}_{12} \mathrm{~S}_{3} \mathrm{Br}_{6}$ (Fig. $1 \mathrm{~b}$ inset), formed well-dispersed long fibers on the $\mathrm{SiO}_{2} / \mathrm{Si}$ substrate (Fig. $1 \mathrm{~b}$ and Fig. S2), which is similar to cases of many small organic molecules. Compared with the XRD data of the source powder, an extra peak located at approximately $9.14^{\circ}$ appeared in the patterns of fibers, indicating that the crystalline structure of the formed fibers differs from that of the bulk samples (Fig. 1c). TEM revealed that the $\pi-\pi$ stacking direction of molecules is along the long axis of the fiber with measured interplanar distances of 1.09 and $0.4 \mathrm{~nm}$ (Fig. 1d, e).

This common vapor-deposition route is not suitable for organizing small $\mathrm{C}_{12} \mathrm{~S}_{3} \mathrm{Br}_{6}$ molecules into $2 \mathrm{D}$ thin films. The process involves the evaporation of small molecules at high temperatures, and such molecules are transported downstream by diffusion and carrier gas flow, resulting in the crystallization of these small molecules on an inert substrate surface at a suitable temperature. In general, increasing the source temperature results in a higher supply of source molecules, and lowering the substrate temperature results in high supersaturation for molecule aggregation. By tuning these factors, the nucleation density, size, and crystallinity of the as-grown organic crystals can be modulated (Fig. S2).

In contrast to an inert substrate, we realized this goal using an active $\mathrm{Cu}$ substrate, which can react with $\mathrm{Br}$ groups within these molecules, providing the possibility of local polymerization of de-brominated $\mathrm{C}_{12} \mathrm{~S}_{3} \mathrm{Br}_{6}$ directly on $\mathrm{Cu}$ substrate. Under a typical vapor-deposition condition, organic materials are formed on polycrystalline $\mathrm{Cu}$ foil surfaces, and the as-grown materials can readily be transferred onto other substrates, such as $\mathrm{SiO}_{2} / \mathrm{Si}$, by electrochemical bubbling, which is widely used in transferring graphene to $\mathrm{Cu}$. The transferred sample shows the morphology of a thin film (Fig. 1f, g). Optical microscopy revealed that the thin film has a relatively uniform, continuous, and distinct color 
a

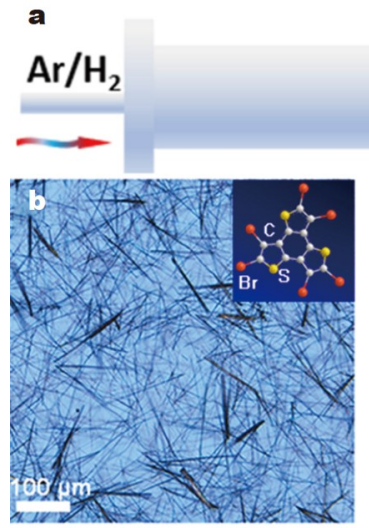

Zone 1
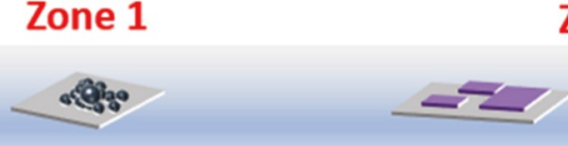

Zone 2
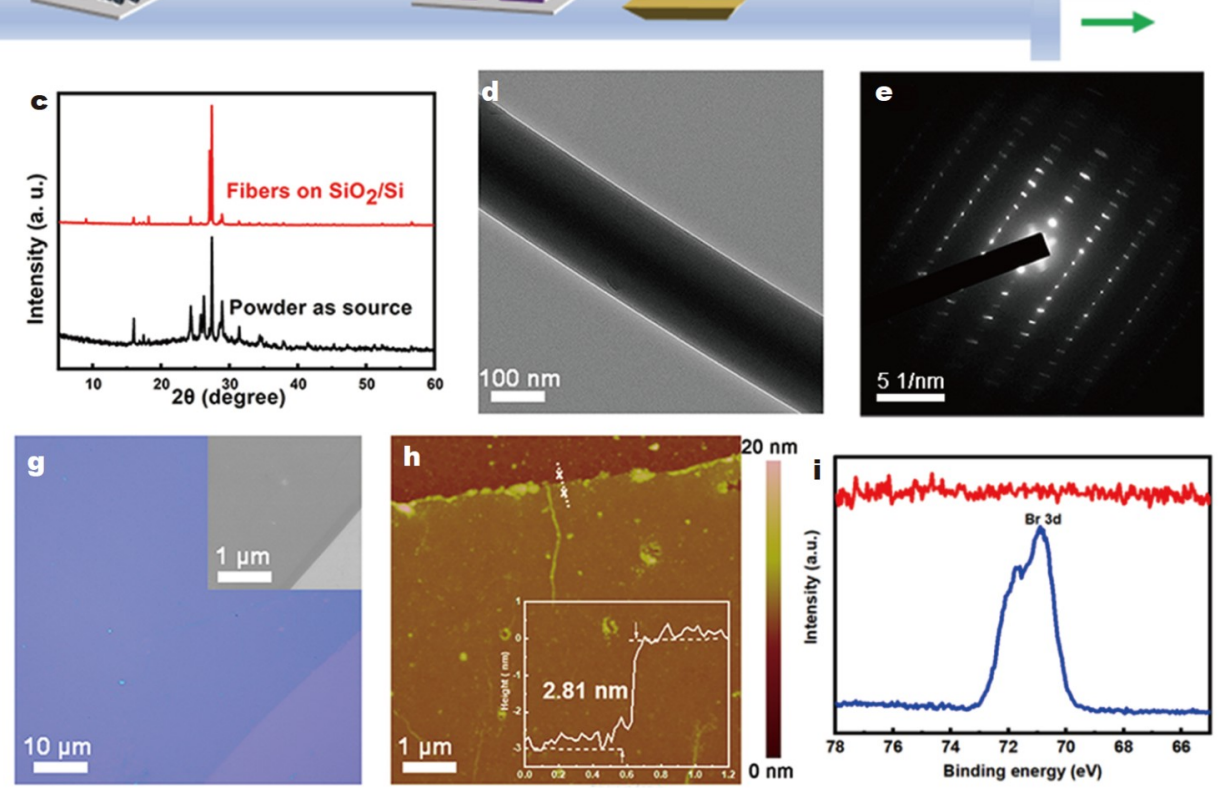

Figure 1 Comparison of the fiber and thin film formed on $\mathrm{SiO}_{2} / \mathrm{Si}$ and $\mathrm{Cu}$ substrates using the same $\mathrm{C}_{12} \mathrm{~S}_{3} \mathrm{Br}_{6} \mathrm{molecule}$ source. (a) $\mathrm{Schematic}$ of vapor deposition process with two heat zones. (b) Scanning electron microscopy (SEM) image of fibers formed on $\mathrm{SiO}_{2} / \mathrm{Si}$ substrate. (c) Comparison of $\mathrm{XRD}$ data for fibers and the source. (d) TEM image of a fiber. (e) Corresponding selected area electron diffraction (SAED) pattern of the fiber in (d). (f) Optical image of thin-film transferred onto a $\mathrm{SiO}_{2} / \mathrm{Si}$ substrate. Inset: a $5 \mathrm{~cm} \times 5 \mathrm{~cm}$ thin film on a $\mathrm{Si} / \mathrm{SiO}_{2}$ wafer. (g) Optical closed-up image of a thin film with an edge. Inset: the corresponding SEM image of the thin film sample. (h) AFM height image of the thin film with an edge on a SiO $2 / \mathrm{Si}$ substrate, which shows that the thickness of the thin film is approximately $2.81 \mathrm{~nm}$ (inset). (i) XPS spectra of a thin film on $\mathrm{SiO}_{2} / \mathrm{Si}$ (top red) and source (bottom blue), showing the absence of Br groups in the thin film.

contrast with respect to the adjacent $\mathrm{SiO}_{2} / \mathrm{Si}$ surface, indicating that this process allows the formation of $2 \mathrm{D}$ thin films from small molecules.

Several techniques were used to characterize the sample. The thickness of the thin films is in the range of $2-5 \mathrm{~nm}$, with a typical value of approximately $3 \mathrm{~nm}$ (Fig. 1h). The area of the thin film is the same as the $\mathrm{Cu}$ area. XPS revealed the absence of detectable $\mathrm{Br}$ signals in the transferred samples, indicating that $\mathrm{Br}$ groups in the as-grown films were removed (Fig. 1i). The Raman spectrum of the sample shows two broad peaks at 1348 and $1543 \mathrm{~cm}^{-1}$ (Fig. S3). The former is similar to the D peak in the graphene sample, which is attributed to the defect-induced phonon mode, and the latter is red-shifted compared with the G peak in an ideal graphene lattice. In addition, the peak broadening reflects the degree of deviation from the perfect longrange periodicity of lattice in the samples.

XRD and TEM were employed to further investigate the microstructure of the samples. The XRD pattern of the sample showed several peaks (Fig. $2 \mathrm{a}$ ). The two main peaks are located at $3.68^{\circ}, 12.12^{\circ}$, and $12.51^{\circ}$, corresponding to $2.40,0.73$, and $0.71 \mathrm{~nm}$ of crystal plane distances, respectively. Other detectable peaks are around $17.18^{\circ}$ and $20.77^{\circ}$, corresponding to a distance of about 0.52 and $0.43 \mathrm{~nm}$. On the other hand, TEM analysis of the transferred samples showed two typical cases. The TEM image of the thinner film with low contrast showed clear lattice fringes (Fig. 2b, c). However, thinner films are vulnerable to the exposure of high-energy electrons during TEM imaging. A periodic lattice structure could be observed in the thinner regions during the initial imaging; however, the patterns trans- ited to an amorphous state too rapidly to capture the image (Fig. S4). On the other hand, other parts of the films showed nonuniform contrast within the thin films, reflecting a small fluctuation of the sample thickness (Fig. 2b, c, and Fig. S4). Further imaging of the darker region showed various kinds of well-defined lattice structures (Fig. S5), and one of the zoom-in regions is shown in Fig. 2d. In addition, typical moiré patterns were observed on the thicker films, reflecting a twist of the upper and lower crystalline layers (Fig. S6).

These complex structural data obtained from XRD and TEM analyses prevented the detailed determination of the sample's microstructure, indicating the polycrystalline nature or structural diversity of the thin films. However, by carefully analyzing the XRD and TEM data simultaneously, we could obtain the microstructural information to some extent at the level of the thin film. First, XRD mainly reveals the out-of-plane distance, whereas TEM images show the in-plane distance due to the geometric configuration of thin films on substrates or TEM grids. Thus, the several out-of-plane distances revealed by XRD (Fig. 2a) indicate that symmetric organic molecules have a tilted orientation with respect to the substrate surface. On this basis, molecules arrange themselves through strong $\pi-\pi$ interactions parallel to the molecule's plane, which is consistent with the TEM results and molecule size. The dominant peaks at small angles in the XRD data also indicate that the stable tilt angles of molecules within thin films are not unique, and thin films consist of small domains (from several to several tens of nanometers) with different tilt angles with respect to the substrate surface. Highly-polycrystalline films are detrimental to electron 

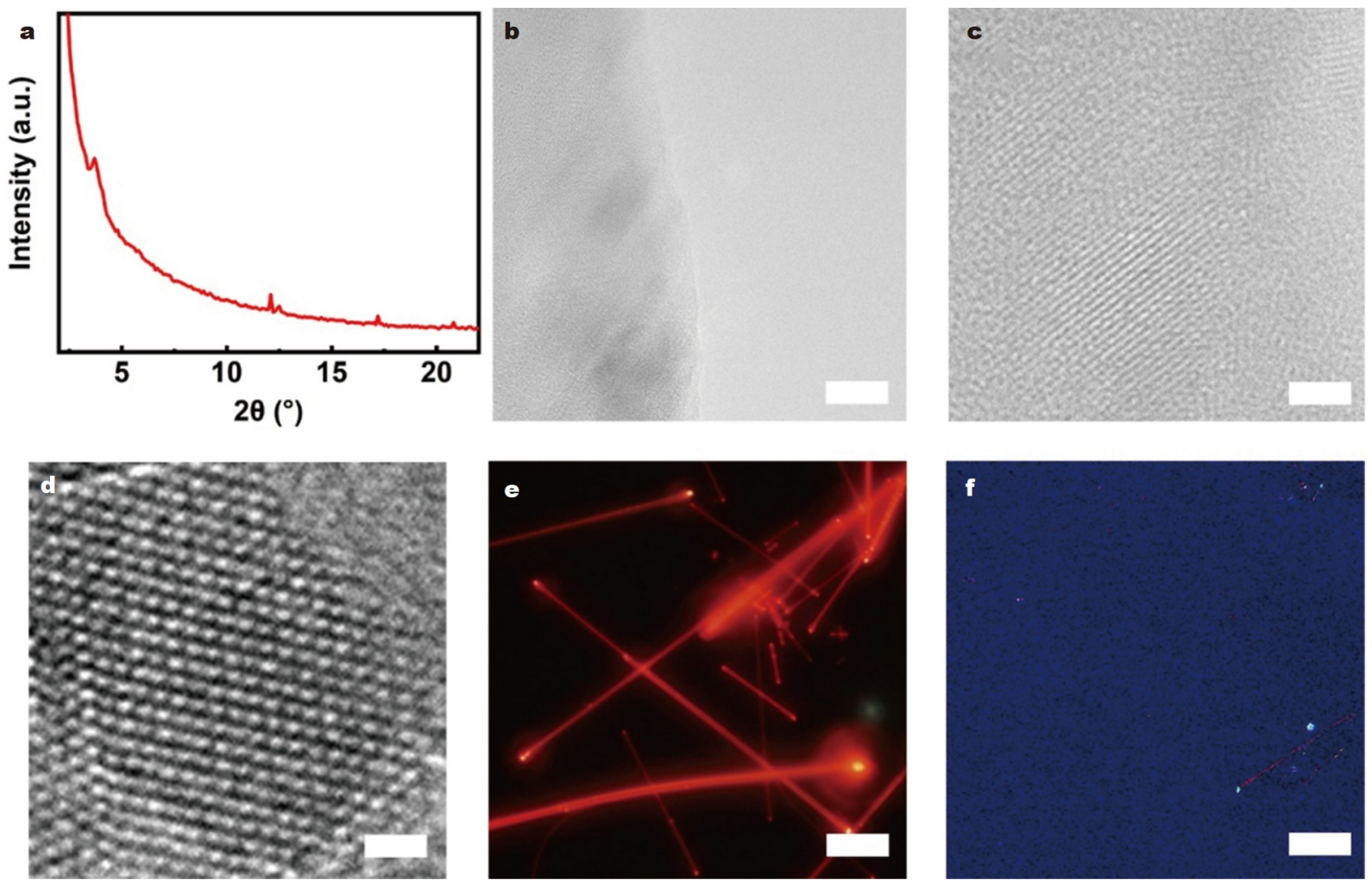

Figure 2 Characterization of samples. (a) XRD patterns of the synthesized thin film. (b) TEM image of the thin film (the scale bar is $5 \mathrm{~nm}$ ). (c) Zoomed-in TEM image showing lattice fringes on the thin film sample (the scale bar is $2 \mathrm{~nm}$ ). (d) TEM image of a thicker area within the thin film (the scale bar is $1 \mathrm{~nm}$ ). (e) Photoluminescence image of fibers on a $\mathrm{SiO}_{2} / \mathrm{Si}$ substrate (the scale bar is $50 \mu \mathrm{m}$ ). (f) Photoluminescence image of a thin film transferred onto a $\mathrm{SiO} / 2 \mathrm{Si}$ substrate (the scale bar is $20 \mu \mathrm{m}$ ).

transport; the realization of a large-area, dimension-controllable polycrystalline organic film distinguishes the thin films obtained from those synthesized through other methods, which are usually amorphous organic films.

In comparison, no such uniform thin organic films were found on the $\mathrm{SiO}_{2} / \mathrm{Si}$ substrate placed closely to the $\mathrm{Cu}$ foil under the same growth conditions. Generally, very few materials were randomly dispersed on the $\mathrm{SiO}_{2} / \mathrm{Si}$ substrate. This observation can be readily explained. Without the ability of debromination of organic molecules by inert substrates, the higher molecule thermal motion at the collecting regions hinders effective nucleation from forming organic crystals. In addition, the microstructure of organic fibers differs from that of the thin films. For example, the peak positions of fibers from XRD data are located at $9.14^{\circ}, 16.04^{\circ}, 18.19^{\circ}$, and others, which differ from those of thin-film, demonstrating two kinds of microstructures for two materials. This difference was confirmed by comparing the TEM results of the two materials (Figs 1d, e, and 2d). Moreover, the fibers exhibited pronounced photoluminescence (Fig. 2e), where the excitation wavelength of 325-375 nm caused the emission of red light by the fibers with a good waveguide property. In contrast, the thin film did not show good photoluminescence (Fig. 2f), possibly due to the polymerization state of the thin film, which changes the electronic structure, thus quenching the light emission.

To further investigate the growth mechanism, the growth of the films at different stages was recorded. Generally, the partially covered thin film is formed on the $\mathrm{Cu}$ surface at the early stage of growth (Fig. 3a), and the optical uniform contrast in Fig. 3a indicates a relatively uniform thickness. The coverage of the film on the $\mathrm{Cu}$ foil increased with the growth time by island growth and merging process (Fig. $3 \mathrm{~b}, \mathrm{c}$ ). In addition, the morphologically distinct partially covered thin films (Fig. 3d) and dynamic growth to full-covered films (Fig. 3e, f) were also observed. The building blocks of the thin film were "aligned" and anisotropic shaped, which is different from islands with no specific geometric shapes in Fig. 3a. Both cases showed the same dynamic growth evolution, indicating that organic thin films follow a common nucleation and growth mechanism.

The difference in the thin-film morphology at different regions was directly correlated with the type of $\mathrm{Cu}$ surface crystal plane. Inset of Fig. $3 \mathrm{~g}$ shows an optical image of the thin film on polycrystalline $\mathrm{Cu}$. Different $\mathrm{Cu}$ planes were separated by obvious grain boundaries. The same area in the transferred sample (Fig. 3g) showed one-to-one correspondence to that on the $\mathrm{Cu}$ surface. Evidently, for a specific $\mathrm{Cu}$ facet, the thin film was homogeneous, and different $\mathrm{Cu}$ facets resulted in films with different thicknesses reflected by optical contrast. Further detailed observations (Fig. $3 \mathrm{~h}, \mathrm{i}$ ) revealed different morphologies and thicknesses for films grown on different facets. These observations are important as they establish a basis for the growth of uniform thin films. Indeed, homemade single-crystal $\mathrm{Cu}(111)$ foils were used to achieve large-area uniform thin films, as shown in Fig. 3b. This $\mathrm{Cu}$-facet-dependent growth explains some observations. For example, first, different tilt configurations of organic molecules revealed that the XRD data are attributed to the different arrangements of molecules on different $\mathrm{Cu}$ lattices, indicating a kind of epitaxy between large 

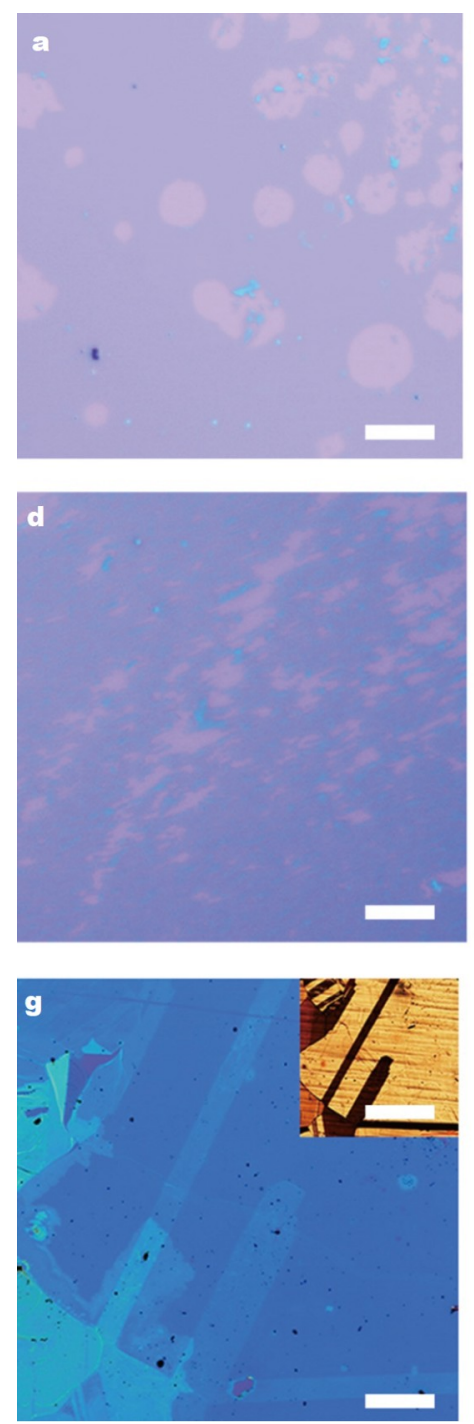
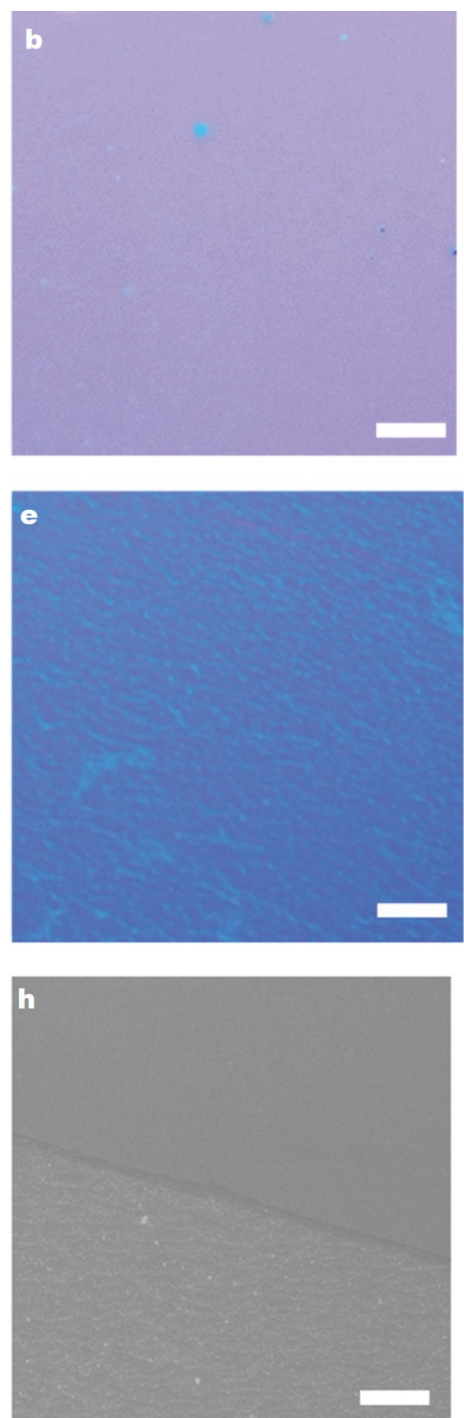
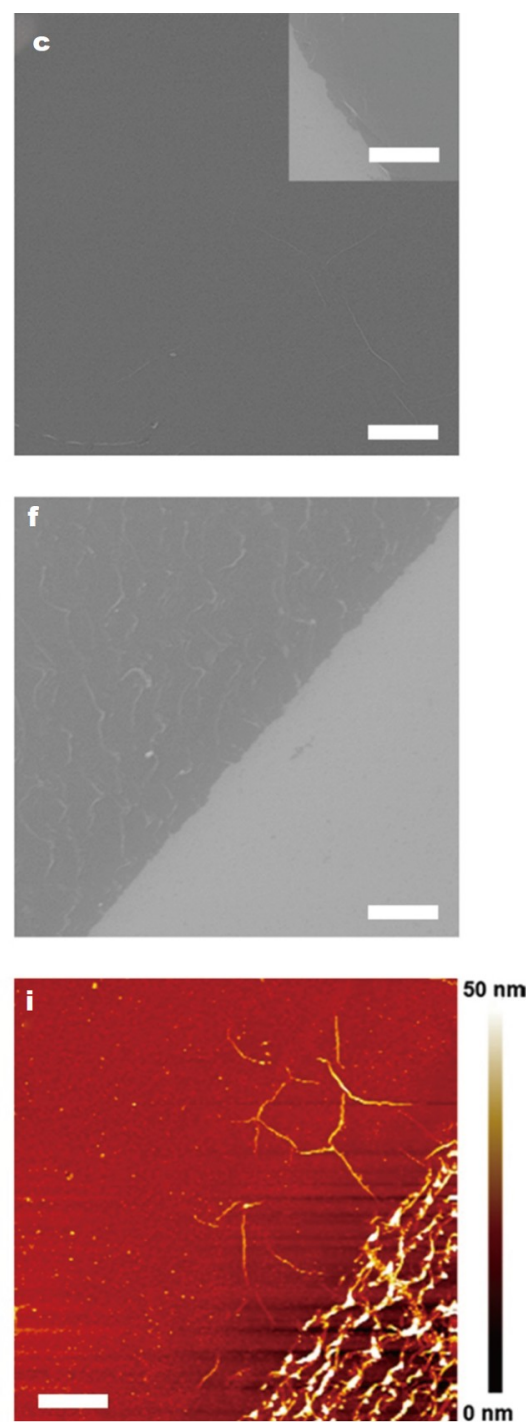

Figure 3 Images of organic films during the growth process. (a) Optical image of a film transferred to a $\mathrm{SiO}_{2}$ substrate without full growth. (b, c) Optical and SEM images of a continuous thin film transferred to a $\mathrm{SiO}_{2} / \mathrm{Si}$ substrate, respectively. The inset of (c) shows the SEM image of the thin film edge. (d) Optical image of a partially grown film on different $\mathrm{Cu}$ facets transferred to $\mathrm{SiO}_{2}$ substrates. (e, f) Optical and SEM images of a continuous thin film transferred to a $\mathrm{SiO}_{2} / \mathrm{Si}$ substrate, respectively. Note that thin film morphology is dependent on the Cu facet. All scale bars in (a-f) are $10 \mu \mathrm{m}$, and the scale bar of the inset of (c) is $1 \mu \mathrm{m}$. (g) Optical image of a thin film grown on polycrystalline $\mathrm{Cu}$ and transferred to a $\mathrm{SiO}_{2} / \mathrm{Si}$ substrate. The inset shows the optical image of the same region of the as-grown thin film on a $\mathrm{Cu}$ surface. The scale bars of (g) and the inset are $200 \mu \mathrm{m}$. (h) SEM image of the interface of thin films grown on two adjacent $\mathrm{Cu}$ grains with distinct morphologies. The scale bar is $10 \mu \mathrm{m}$. (i) AFM image of the interface similar to (h). Certain similar features are observed in (h) and (i). The scale bar is $15 \mu \mathrm{m}$.

organic molecules and $\mathrm{Cu}$ lattices rarely found in organic growing systems. Second, in addition to the role of the $\mathrm{Cu}$ facet template, $\mathrm{Cu}$ also plays a role in reacting with $\mathrm{Br}$ groups. Experiments showed that the thin film on $\mathrm{Cu}(111)$ grew slower than other facets, which is consistent with its smallest surface energy compared with that of other facets.

Several parameters, such as temperature, growth time, pressure, carrier gas, and $\mathrm{Cu}$ crystal plane, could modulate the growth process. The growth of the thin film on the $\mathrm{Cu}$ surface could be realized in a wide window of experimental conditions. For example, the source and substrate temperatures were varied from 180 to $340^{\circ} \mathrm{C}$ and 280 to $340^{\circ} \mathrm{C}$, respectively. In general, the higher temperature and shorter distance between the source and the $\mathrm{Cu}$ foil resulted in faster growth of the thin film. Especially, the continuous thin film formed within a few minutes. The carrier gas composition ( $\mathrm{Ar}$ and $\mathrm{H}_{2}$ ) and pressure showed no dramatic impact on the growth results.

Our approach for organic thin-film syntheses can be used for several aspects different from other methods. For example, solution-based methods work only for materials that have good solubility with solvents. In contrast, our approach has no such limitations. It can be applied to other molecules. Two molecules of $\mathrm{C}_{24} \mathrm{H}_{4} \mathrm{O}_{2} \mathrm{Br}_{2}$ and $\mathrm{C}_{24} \mathrm{H}_{12} \mathrm{Br}_{2} \mathrm{~N}_{4}$ formed an equivalence class with $\mathrm{C}_{12} \mathrm{~S}_{3} \mathrm{Br}_{6}$, in which they share the same $\pi$-conjugated structure and $\mathrm{Br}$ groups but with different molecule sizes, shapes, and $\mathrm{Br}$ numbers. The TGA results of the two molecules are shown in Fig. S7. Interestingly, both $\mathrm{C}_{24} \mathrm{H}_{4} \mathrm{O}_{2} \mathrm{Br}_{2}$ and $\mathrm{C}_{24} \mathrm{H}_{12} \mathrm{Br}_{2} \mathrm{~N}_{4}$ molecules formed a similar thin film using this approach, as shown in Fig. $4 \mathrm{a}-\mathrm{c}$ and $\mathrm{d}-\mathrm{f}$, respectively. As a demonstration, no obvious peaks were detected in the XRD test 

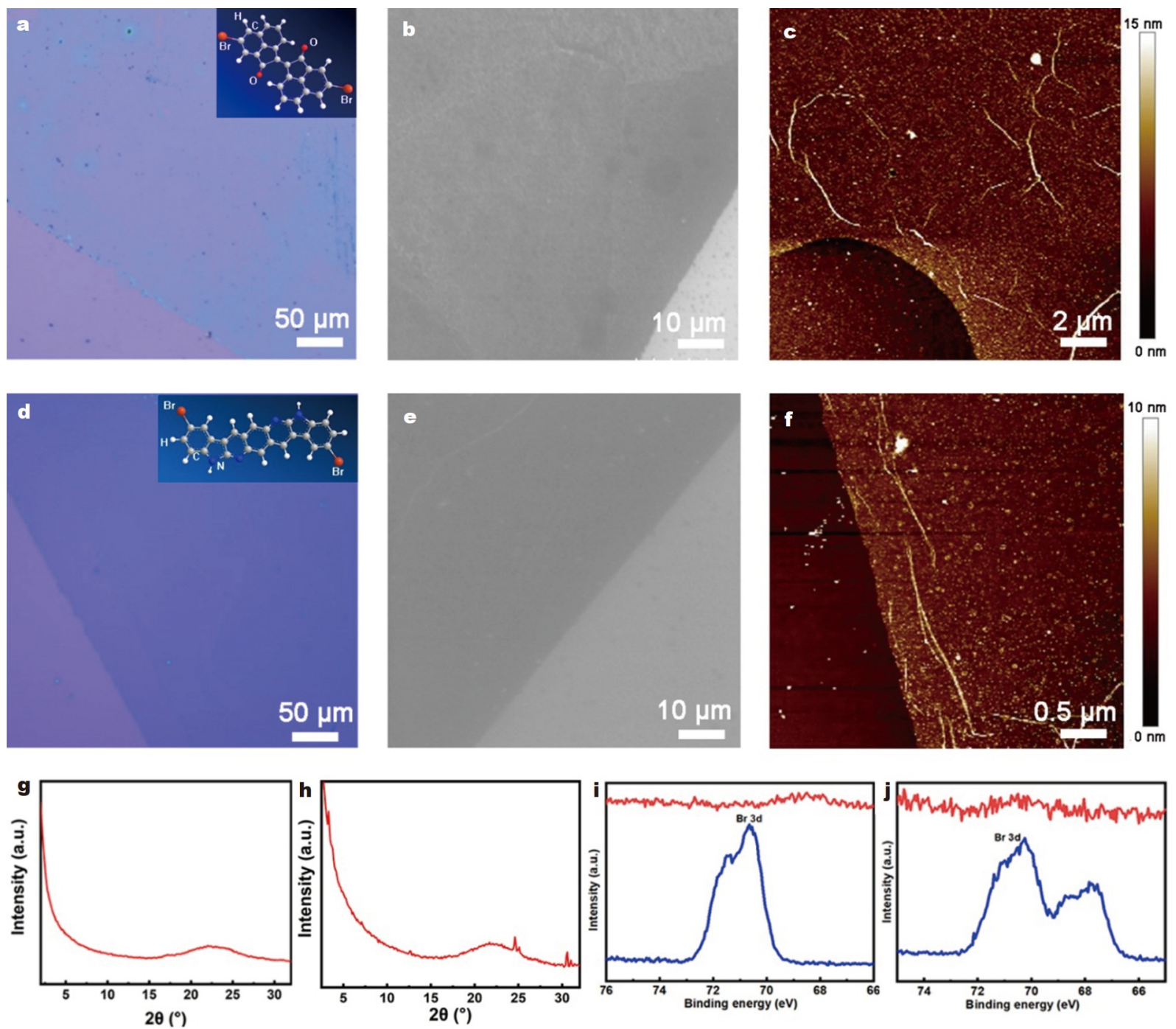

Figure 4 Growth of a thin film with other conjugated molecules containing Br groups. (a-c) Optical, SEM, and AFM images, respectively, of a grown film transferred to a $\mathrm{SiO}_{2} / \mathrm{Si}$ substrate using $\mathrm{C}_{24} \mathrm{H}_{4} \mathrm{O}_{2} \mathrm{Br}_{2}$ as the source. The inset in (a) shows the molecule structure of the sample. (d-f) Optical, SEM, and AFM images, respectively, of a grown film transferred to a $\mathrm{SiO}_{2} / \mathrm{Si}$ substrate using $\mathrm{C}_{24} \mathrm{H}_{12} \mathrm{Br}_{2} \mathrm{~N}_{4}$ as the source. The inset in (d) shows the molecule structure of the sample. (g, h) XRD spectra of thin films synthesized from $\mathrm{C}_{24} \mathrm{H}_{4} \mathrm{O}_{2} \mathrm{Br}_{2}$ and $\mathrm{C}_{24} \mathrm{H}_{12} \mathrm{Br}_{2} \mathrm{~N}_{4}$, respectively. (i, $j$ ) XPS spectra, showing no $\mathrm{Br}$ groups in the corresponding thin films from $\mathrm{C}_{24} \mathrm{H}_{4} \mathrm{O}_{2} \mathrm{Br}_{2}$ (blue) and $\mathrm{C}_{24} \mathrm{H}_{12} \mathrm{Br}_{2} \mathrm{~N}_{4}$ (blue), respectively.

of $\mathrm{C}_{24} \mathrm{H}_{4} \mathrm{O}_{2} \mathrm{Br}_{2}$ (Fig. 4g), and $\mathrm{C}_{24} \mathrm{H}_{12} \mathrm{Br}_{2} \mathrm{~N}_{4}$ also showed several peaks at 3.42, 24.6, 25.1, and 30.6 in XRD tests (Fig. 4h), confirming the formation of a polycrystalline film. Similar to the case of $\mathrm{C}_{12} \mathrm{~S}_{3} \mathrm{Br}_{6}, \mathrm{Br}$ groups in the as-formed films were also removed, as shown in Fig. 4i, j. Based on these results, we compared the growth behavior of our three molecules and previously reported hexabromo-benzene. It has been reported that hexabromo-benzene forms graphene on $\mathrm{Cu}$ by the CVD method [19]. In this case, molecules are polymerized along the molecule plane, minimizing $\pi-\pi$ interactions among molecules in the final product. In contrast, the other three molecules formed a thin film prone to tilting with molecule planes, thus involving strong $\pi-\pi$ interactions for shaping the structure and morphology of as-grown materials. These results are attributed to the molecule size and number of $\mathrm{Br}$ groups in molecules. Qualitatively, the more the $\mathrm{Br}$ groups in molecules, the more the chances for polymerization. On the other hand, larger $\pi$-conjugated molecules tend to have larger $\pi-\pi$ interaction. This analogy provides a picture to understand the various growth behavior of different molecules.

Our approach provides a feasible means for fabricating heterostructures of organic 2D materials [27-29]. As a demonstration, $\mathrm{C}_{12} \mathrm{~S}_{3} \mathrm{Br}_{6} /$ graphene was prepared. Domains or films of graphene are first grown on $\mathrm{Cu}(111)$ surface, followed by organic thin-film growth. Fig. 5a shows a typical optical image of transferred $\mathrm{C}_{12} \mathrm{~S}_{3} \mathrm{Br}_{6}$ /graphene samples. Organic thin-film and graphene parts can be easily identified using their color contrast with respect to the surroundings. It has been proven that organic thin films grow on graphene. First, the morphology of organic thin-film surfaces in the optical image reflects the simple organic film features without the convolution signal with graphene. AFM showed that the height of the graphene and thin films are about 1.6 and $2.5 \mathrm{~nm}$ (Fig. 5b, c), respectively. Second, imaging the randomly peeled organic thin-film/graphene sample confirmed that the organic thin film was grown on top of the graphene (Fig. 5d). Third, Fig. 5e, f compared Raman spectra of 

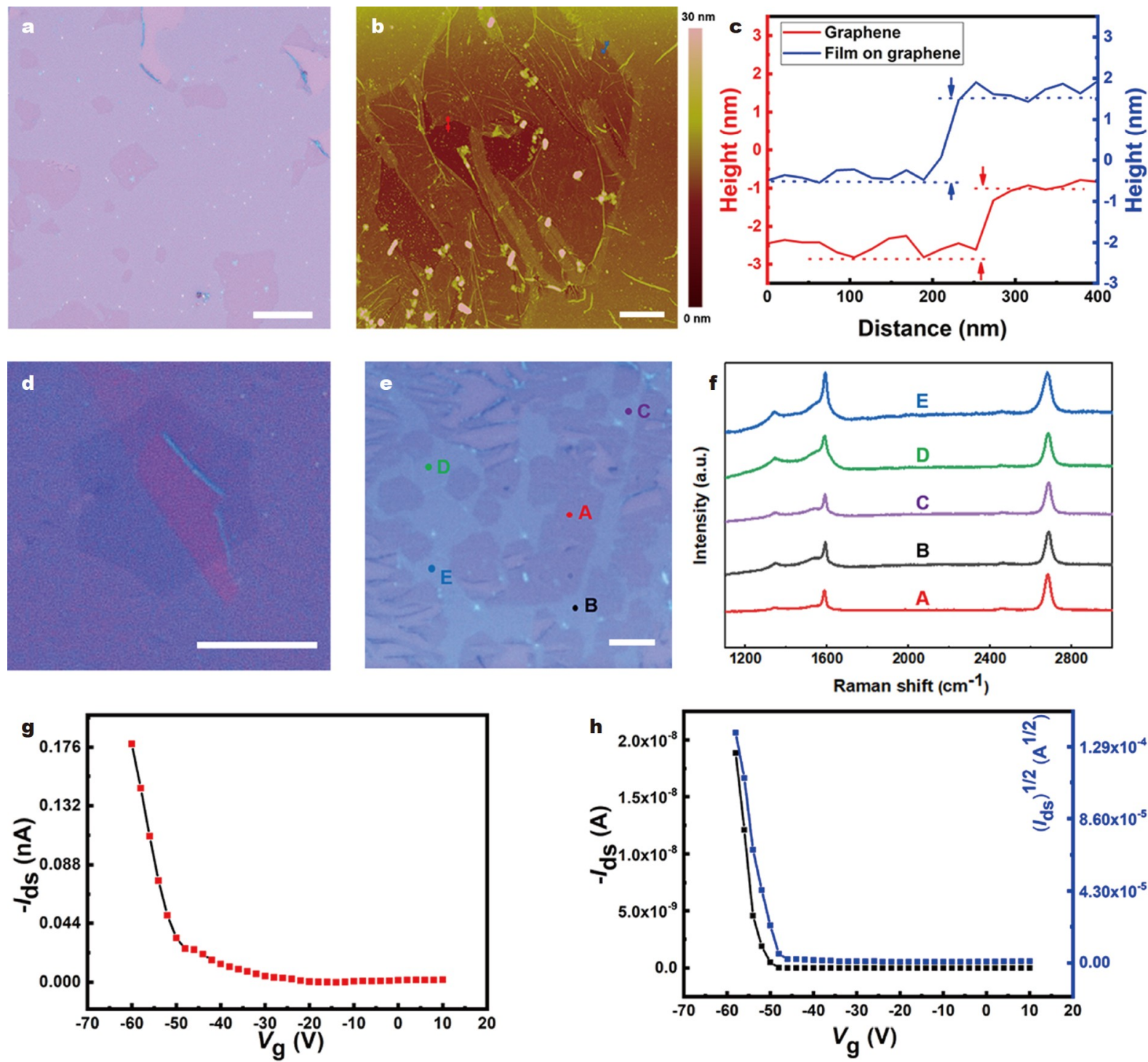

Figure 5 Fabrication of heterostructures. (a) Optical image of a $\mathrm{C}_{12} \mathrm{~S}_{3} \mathrm{Br}_{6}$-derived film/graphene heterostructure transferred to a $\mathrm{SiO}_{2} / \mathrm{Si}$ substrate $($ the scale bar is $10 \mu \mathrm{m}$ ). (b) AFM image of the corresponding heterostructure. Note that the central region with low height consists mainly of graphene (the scale bar is $2 \mu \mathrm{m}$ ). (c) Section analysis of (b), showing the thicknesses of graphene and thin film. (d) Optical image of thin organic films grown on graphene. Note that thin film was peeled, and the underlying graphene on a $300-\mathrm{nm} \mathrm{SiO}_{2} / \mathrm{Si}$ substrate can be identified by its color (purple). The scale bar is $10 \mu \mathrm{m}$. (e) Optical image of a heterostructure with several points labeled for Raman characterization (the scale bar is $10 \mu \mathrm{m}$ ). (f) Corresponding Raman spectra of the labeled points in (e). (g, h) Transfer curves of a field-effect transistor of $\mathrm{C}_{12} \mathrm{~S}_{3} \mathrm{Br}_{6}$-derived thin film and fiber samples, respectively.

graphene and film/graphene. In the case of the film/graphene, despite the absence of graphene on the surface in the optical image, the corresponding Raman spectra reveal the superposition of the individual spectrum of each material, confirming the existence of graphene under the organic thin film.

Forming an organic thin film on graphene is intriguing as there should be a route for $\mathrm{Cu}$ atoms under the graphene layer to transport. There was no statistical difference in thin-film growth on mono- or bi-layer graphene. Despite this, the transparency of graphene to catalysis of the underlying $\mathrm{Cu}$ has been previously reported, and there are many practical routes for $\mathrm{Cu}$ transport through grain boundaries, defects, wrinkles, or exposed areas [30]. On the other hand, at present, crystal sizes of thin films are relatively small, which compromises their electronic transport, resulting in an on-off ratio of $\sim 94$ and measured mobility of $10^{-4} \mathrm{~cm}^{2} \mathrm{~V}^{-1} \mathrm{~s}^{-1}$ (Fig. $5 \mathrm{~g}$ ), which are lower than those of the corresponding fiber (on-off ratio of $\sim 10^{4}$ and mobility of $0.53 \mathrm{~cm}^{2} \mathrm{~V}^{-1} \mathrm{~s}^{-1}$, Fig. 5h). Further optimization of conditions or post-engineering material crystallinity is expected to improve the properties of these thin films. Indeed, post-thermal annealing of samples can increase crystal sizes up to several tens of nanometers.

\section{CONCLUSIONS}

In summary, we demonstrate the growth of wafer-scale polymerized organic ultrathin films through $\mathrm{Cu}$ surface-mediated reaction and polymerization of several equivalent $\mathrm{Br}$-containing $\pi$-conjugated small molecules. This method is useful for conjugated molecules with poor solvent solubility and can be applied to several similar materials containing Br groups. Taking the advantage of $\mathrm{Cu}$ crystal facet-dependent growth, this method allows the synthesis of large-scale, ultrathin, and polycrystalline 
organic films with uniform thicknesses. Easy synthesis of organic thin-film and 2D heterostructures gives room for various functional materials and device applications.

\section{Received 29 October 2021; accepted 1 December 2021;} published online 21 January 2022

1 Tada H, Saiki K, Koma A. Structural analysis of lead phthalocyanine ultrathin films grown on cleaved faces of alkali halides by reflection high energy electron diffraction. Surf Sci, 1992, 268: 387-396

2 Xi M, Bent BE. Mechanisms of the Ullmann coupling reaction in adsorbed monolayers. J Am Chem Soc, 1993, 115: 7426-7433

3 Bao Z, Lovinger AJ, Dodabalapur A. Organic field-effect transistors with high mobility based on copper phthalocyanine. Appl Phys Lett, 1996, 69: 3066-3068

4 Yanagi H, Morikawa T. Self-waveguided blue light emission in $p$ sexiphenyl crystals epitaxially grown by mask-shadowing vapor deposition. Appl Phys Lett, 1999, 75: 187-189

5 Menard E, Podzorov V, Hur SH, et al. High-performance n- and p-type single-crystal organic transistors with free-space gate dielectrics. Adv Mater, 2004, 16: 2097-2101

6 Blake MM, Nanayakkara SU, Claridge SA, et al. Identifying reactive intermediates in the Ullmann coupling reaction by scanning tunneling microscopy and spectroscopy. J Phys Chem A, 2009, 113: 13167-13172

7 Sundar VC, Zaumseil J, Podzorov V, et al. Elastomeric transistor stamps: Reversible probing of charge transport in organic crystals. Science, 2004, 303: 1644-1646

8 Lee HM, Moon H, Kim HS, et al. Abrupt heating-induced high-quality crystalline rubrene thin films for organic thin-film transistors. Org Electron, 2011, 12: 1446-1453

9 Oyamada $\mathrm{T}$, Uchiuzou $\mathrm{H}$, Akiyama $\mathrm{S}$, et al. Lateral organic lightemitting diode with field-effect transistor characteristics. J Appl Phys, 2005, 98: 074506

10 Liu J, Jiang L, $\mathrm{Hu} \mathrm{W}$, et al. Monolayer organic field-effect transistors. Sci China Chem, 2019, 62: 313-330

$11 \mathrm{Xu} X$, Yao Y, Shan B, et al. Electron mobility exceeding $10 \mathrm{~cm}^{2} \mathrm{~V}^{-1} \mathrm{~s}^{-1}$ and band-like charge transport in solution-processed $n$-channel organic thin-film transistors. Adv Mater, 2016, 28: 5276-5283

12 Zhang X, Mao J, Deng W, et al. Precise patterning of laterally stacked organic microbelt heterojunction arrays by surface-energy-controlled stepwise crystallization for ambipolar organic field-effect transistors. Adv Mater, 2018, 30: 1800187

13 Lin Y, Huang Z, Wen X, et al. Steering effect of bromine on intermolecular dehydrogenation coupling of poly( $p$-phenylene) on $\mathrm{Cu}(111)$. ACS Nano, 2020, 14: 17134-17141

14 Shu Z, Zhang Q, Zhang P, et al. Preparing two-dimensional crystalline conjugated polymer films by synergetic polymerization and self-assembly at air/water interface. Polym Chem, 2020, 11: 1572-1579

15 Wang L, Zhang X, Dai G, et al. High-mobility air-stable n-type fieldeffect transistors based on large-area solution-processed organic singlecrystal arrays. Nano Res, 2017, 11: 882-891

16 Zhang Y, Jie J, Sun Y, et al. Precise patterning of organic single crystals via capillary-assisted alternating-electric field. Small, 2017, 13: 1604261

17 Yang L, Wei DC. Semiconducting covalent organic frameworks: A type of two-dimensional conducting polymers. Chin Chem Lett, 2016, 27: 1395-1404

18 Wang $\mathrm{C}, \mathrm{Fu} \mathrm{B}$, Zhang X, et al. Solution-processed, large-area, twodimensional crystals of organic semiconductors for field-effect transistors and phototransistors. ACS Cent Sci, 2020, 6: 636-652

19 Jiang L, Niu T, Lu X, et al. Low-temperature, bottom-up synthesis of graphene via a radical-coupling reaction. J Am Chem Soc, 2013, 135: 9050-9054

20 He D, Zhang Y, Wu Q, et al. Two-dimensional quasi-freestanding molecular crystals for high-performance organic field-effect transistors. Nat Commun, 2014, 5: 5162

21 Evans AM, Parent LR, Flanders NC, et al. Seeded growth of singlecrystal two-dimensional covalent organic frameworks. Science, 2018, 361: $52-57$
22 Liu K, Qi H, Dong R, et al. On-water surface synthesis of crystalline, few-layer two-dimensional polymers assisted by surfactant monolayers. Nat Chem, 2019, 11: 994-1000

23 Zhong Y, Cheng B, Park C, et al. Wafer-scale synthesis of monolayer two-dimensional porphyrin polymers for hybrid superlattices. Science, 2019, 366: 1379-1384

24 Toh CT, Zhang H, Lin J, et al. Synthesis and properties of free-standing monolayer amorphous carbon. Nature, 2020, 577: 199-203

25 Yao J, Zhang Y, Tian X, et al. Layer-defining strategy to grow twodimensional molecular crystals on a liquid surface down to the monolayer limit. Angew Chem Int Ed, 2019, 58: 16082-16086

26 Feng G, Jiang J, Zhao $Y$, et al. A sub-10 nm vertical organic/inorganic hybrid transistor for pain-perceptual and sensitization-regulated nociceptor emulation. Adv Mater, 2020, 32: 1906171

27 Liu X, Luo X, Nan H, et al. Epitaxial ultrathin organic crystals on graphene for high-efficiency phototransistors. Adv Mater, 2016, 28: 5200-5205

28 Han J, Wang J, Yang M, et al. Graphene/organic semiconductor heterojunction phototransistors with broadband and bi-directional photoresponse. Adv Mater, 2018, 30: 1804020

29 Yao W, Sun J, Chen J, et al. Controllable preparation and photoelectric applications of two-dimensional in-plane and van der Waals heterostructures. Acta Phys Sin, 2021, 70: 027901

30 Wang M, Kim M, Odkhuu D, et al. Catalytic transparency of hexagonal boron nitride on copper for chemical vapor deposition growth of largearea and high-quality graphene. ACS Nano, 2014, 8: 5478-5483

Acknowledgements This work was supported by the National Basic Research Program of China (2016YFA0200101), the National Natural Science Foundation of China (21633012 and 61890940), the Strategic Priority Research Program of the Chinese Academy of Sciences (CAS) (XDB30000000), and the CAS Key Research Program of Frontier Sciences (QYZDY-SSWSLH029).

Author contributions Liu Y supervised the project. Liu Y and $\mathrm{Wu} B$ designed the experiments and research; Yao W and Yang H performed the experiments; Zhang Q and Shi L provided molecules. Wu B and Yao W wrote the paper. All authors contributed to the general discussion.

Conflict of interest The authors declare that they have no conflict of interest.

Supplementary information Supporting data are available in the online version of the paper.

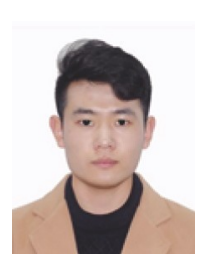

Wenqian Yao received double BSc degrees from Qingdao University of Science and Technology in polymer materials and economics. In 2017, he became a graduate and doctoral student of Sino-Danish College, University of Chinese Academy of Sciences, under the direction of Prof Yunqi Liu and Prof. Bin Wu. He received his master's degree from the University of Copenhagen in 2020. His research interest includes the preparation of $2 \mathrm{D}$ materials and their optical and electrical applications.

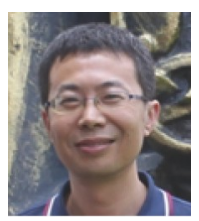

Bin Wu received his BSc degree in 1994 from Inner Mongolia University, China, and $\mathrm{PhD}$ degree in physical chemistry from Peking University in 2002. He joined the Institute of Chemistry, Chinese Academy of Sciences (ICCAS), as an associate professor in 2008 after conducting his postdoctoral research for several years. Currently, he is a professor at ICCAS. His research interest includes the controlled growth of $2 \mathrm{D}$ materials and their electronic applications. 


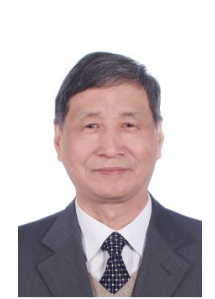

Yunqi Liu is a professor at ICCAS. He graduated from Nanjing University in 1975 and received a doctorate degree from Tokyo Institute of Technology, Japan, in 1991. He was selected as an Academician of CAS in 2015 and a member of the Third World Academy of Sciences in 2018. His research interest includes molecular materials and devices, the synthesis and applications of carbon nanomaterials, and organic electronics.

\section{气固界面聚合反应制备晶圆级均匀超薄二维有机薄膜}

姚文乾 ${ }^{1,2 \dagger}$, 杨贺 ${ }^{1 \dagger}$, 张卿菘 ${ }^{1}$, 施龙献 ${ }^{1,2}$, 孙健哲 ${ }^{1}$, 郭云龙 ${ }^{1}$, 江浪 $^{1}$, 武斌 $1^{*}$, 刘云圻 ${ }^{1,2^{*}}$

摘要 本文介绍了一种利用气固界面聚合反应制备大面积均匀超薄二 维有机分子薄膜的新进展. 通常有机分子薄膜的制备多借助于溶液 法、旋涂法等技术, 所制备的薄膜尺寸、均匀性和厚度可控性较差. 传 统的化学气相沉积法在大面积石墨烯、氮化硼、过渡金属硫族化合物 等二维材料的制备方面应用广泛，但在有机分子薄膜制备领域却鲜有 报道. 本文中, 我们展示了一种基于化学气相沉积生长连续、均匀、超 薄(2-5 nm) 的晶圆级多晶有机薄膜的一般方法. 该方法基于铜表面催 化的一系列含溴 $\pi$-共轭小分子的聚合, 其中局部表面聚合和有机分子 内部 $\pi-\pi$ 相互作用决定了薄膜的尺寸和均匀性, 研究表明薄膜的生长形 貌和速度与铜晶面的类型密切相关, 基于此在 $\mathrm{Cu}(111)$ 表面获得了更为 均匀的薄膜. 通过另外两种有机小分子薄膜的制备有效地证明了该方 法的可拓展性. 该方法为基于难溶性有机小分子可控制备大面积原子 厚度薄膜和相关异质结构, 如有机膜/石墨烯, 及其他功能材料和器件 提供了新空间. 